\title{
Irrepressible pain treatment in a subject treated with posterior vertebral arthrodesis for a chest lumbar idiopathic scoliosis
}

\author{
Marianna Chierchia, ${ }^{1}$ Carolina Baiano, ${ }^{2}$ Alfredo Romeo, ${ }^{2}$ Dante Ronca ${ }^{1}$ \\ ${ }^{1}$ Multidisciplinary Department of Medical, Surgical and Dental Specialties, Second Orthopedic \\ Clinic, Second University of Naples; ${ }^{2}$ Department of Physical Medicine and Rehabilitation, \\ Umberto I Hospital, Siracusa, Italy
}

\begin{abstract}
We present a case of a 56-year old woman with chest lumbar idiopathic scoliosis treated with an oxygen-ozone therapy cycle $(10-\mu \mathrm{g}$ paravertebral and $3-\mu \mathrm{g}$ subcutaneous infiltrations) for irrepressible pain, as a consequence of a vertebral posterior arthrosis surgery.
\end{abstract}

\section{Case Report}

In 2011, a 56-year old women affected by chest-lumbar idiopathic scoliosis $68^{\circ}$ Coob (Figure 1) underwent a vertebral posterior arthrosis surgery from T6 to L5 (Figure 2).

In August 2012, the patient presented to our Department with a drug-resistant left sciatic nerve inflammation and functional limitation; she was administered gabapentin, anti-inflammatory drugs, opioid pain-reliever, but the treatment was unsuccessful.

In September 2012 she started an oxygen-ozone therapy cycle with $10 \mu \mathrm{g}$ paravertebral infiltrations and $3 \mu \mathrm{g}$ subcutaneous infiltrations.

In November 2012, pain symptom disappeared and functionality restored.

Correspondence: Marianna Chierchia, Multidisciplinary Department of Medical, Surgical and Dental Specialties, Second Orthopedic Clinic, Second University of Naples, via Luigi De Crecchio 6, 80130 Naples, Italy.

E-mail: mariannachierchia@virgilio.it

Key words: Pain treatment; posterior vertebral arthrodesis; chest lumbar idiopathic scoliosis.

Received for publication: 12 January 2016.

Accepted for publication: 24 February 2016.

(C) Copyright M. Chierchia et al., 2016

Licensee PAGEPress, Italy

Ozone Therapy 2016; 1:5839

doi:10.4081/ozone.2016.5839

This article is distributed under the terms of the Creative Commons Attribution Noncommercial License (by-nc 4.0) which permits any noncommercial use, distribution, and reproduction in any medium, provided the original author(s) and source are credited.

\section{Discussion and Conclusions}

The term scoliosis refers to an abnormal curve in the spine in three main planes: frontal, horizontal and sagittal planes. It occurs with an enduring morphological deformation of the spine itself (structuring). Among all the kinds of scoliosis the most common one is the idiopathic one, with a prevalence of $8 \%$. An early diagnosis becomes essential in order to prevent the pathology to worsen, ending up with a surgical intervention.

Vertebral stabilization surgeries include posterior vertebral arthrodesis, which may also involve complications. Motion proximal adjoining segments complications are uncommon in literature; only one $\mathrm{C6}-\mathrm{C} 7$ subluxation case has been noticed in a $\mathrm{T} 1$ proximally extended arthrodesis (Table 1). ${ }^{1}$

Complications observed in terms of arthrodesis distal movement segments are very common and they are represented by functional overload and the degenerative phenomenon due to that. X-rays of patients who underwent a surgery from over 10 years showed traction osteophytes with a disc height reduction in 1/4 cases. There is a clear arthrosis interapofisarias in $12 \%$ of cases, but a symptomatic vertebral canal stenosis never showed. In the Italian experience there is a retrolisthesis of the last vertebrae, which underwent an arthrodesis, only in $25 \%$ of patient at L4-L5 level. It is much rarer at L5-S1 level and proximal at $\mathrm{L} 4,{ }^{2}$ even if in literature have been reported frequencies up to $81 \%{ }^{3}$ Although the evidence of those signs, no correlation between traction osteophytes, discopathy, arthrosis interapofisarias, retrolisthesis and low back pain symptoms was found. Nor spondylosis or spondylolisthesis have been noticed at the lower limit of the arthodesis, thus confirming how rare are those kind of complications. ${ }^{4-6}$ It is important not to over scrape the last vertebrae laminae treated with arthrodesis, in order not to weaken it, especially at L 4 or L5 level. It was noticed, at sacroiliac articulation level, a clear arthrosis, in $30 \%$ of patients 10 years after surgery. No pain symptoms were attributed to that.

Arthrodesis area adjoining segments overload symptoms prevention is accomplished by limiting the fusion of the area strictly needed, especially regarding the mobile distal segments, in strict accordance with placing the hook in stable position, only including the vertebrae of primary curves and avoiding non-structural compensation curves arthrodesis. $^{7}$

In vertebral arthrodesis with Harrington's distractor, an autologous bone graft coming from the iliac wing mono or bilaterally is almost constantly used. The posterior surface of the ileum is normally reached by a second transverse linear incision practiced at the height of the upper posterior iliac spine, most frequently on the left side. The seat of sampling, in arthrodesis extended to lower lumbar trait, is reached by a subcutaneous main incision. In remote check up, a pain subsequent to local palpation was noticed, but only in $4 \%$ of cases there was 
